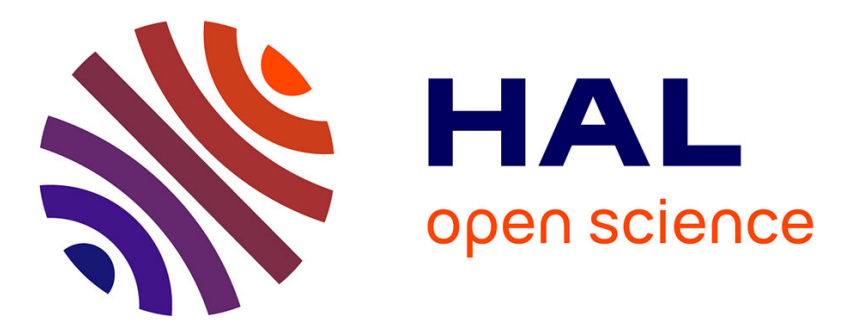

\title{
Écoulement rhéofluidifiant dans un coude et une bifurcation plane symétrique. Application à l'écoulement sanguin dans la grande circulation
}

Marc Thiriet, G. Martin-Borret, Frédéric Hecht

\section{- To cite this version:}

Marc Thiriet, G. Martin-Borret, Frédéric Hecht. Écoulement rhéofluidifiant dans un coude et une bifurcation plane symétrique. Application à l'écoulement sanguin dans la grande circulation. Journal de Physique III, 1996, 6 (4), pp.529-542. 10.1051/jp3:1996139 · jpa-00249475

HAL Id: jpa-00249475

https://hal.science/jpa-00249475

Submitted on 1 Jan 1996

HAL is a multi-disciplinary open access archive for the deposit and dissemination of scientific research documents, whether they are published or not. The documents may come from teaching and research institutions in France or abroad, or from public or private research centers.
L'archive ouverte pluridisciplinaire HAL, est destinée au dépôt et à la diffusion de documents scientifiques de niveau recherche, publiés ou non, émanant des établissements d'enseignement et de recherche français ou étrangers, des laboratoires publics ou privés. 


\title{
Écoulement rhéofluidifiant dans un coude et une bifurcation plane symétrique. Application à l'écoulement sanguin dans la grande circulation
}

\author{
M. Thiriet $\left({ }^{1,2, *}\right)$, G. Martin-Borret $\left({ }^{1}\right)$ et F. Hecht $\left({ }^{1}\right)$ \\ ( $\left.{ }^{1}\right)$ INRIA, Rocquencourt, BP 105, 78153 Le Chesnay Cedex, France \\ $\left({ }^{2}\right)$ LBHP $\left({ }^{* *}\right)$, case 7056, UP7, 75251 Paris Cedex 05, France
}

(Reçu le 9 octobre 1995, révisé le 14 décembre 1995, accepté le 10 janvier 1996)

PACS.87.10.+e - General theoretical and mathematical biophysics

PACS.87.45.Ft - Rheology of body fluids

\begin{abstract}
Résumé. - Des simulations numériques d'écoulement stationnaire laminaire de fluide, dont le comportement rhéologique est rhéofluidifiant, ont été effectuées dans un coude (angle de courbure de 90 degrés, rapport de courbure de 1/10) et une bifurcation plane symétrique (angle de bifurcation de 70 degrés, rapport des sections de 0,8). La méthode des éléments finis a été employée. Le fluide est supposé suivre un comportement newtonien généralisé, représenté par la loi de Carreau, adaptée aux faits d'observation. Les champs de grandeurs hydrodynamiques sont comparés aux champs obtenus lorsque le comportement est newtonien. Tout modèle d'écoulement de sang doit, a priori, prendre en compte le comportement non-newtonien du fluide. Toutefois, cet impératif est discuté lorsque l'investigation porte sur les gros troncs artériels.
\end{abstract}

\begin{abstract}
A numerical study of steady laminar flow has been performed in a bend (curvature angle of 90 degrees, curvature ratio of $1 / 10$ ) and in a symmetrical planar bifurcation (bifurcation angle of 70 degrees, area ratio of 0.8 ) using a finite element method. The fluid is assumed to be incompressible. Its shear-thinning behaviour is modelled by a generalized Newtonian constitutive law, described by the Carreau model. The fields of the involved physical quantities are compared to those obtained with a Newtonian fluid. The non-Newtonian blood flow is strongly different from the flow computed with the usual viscosity of the blood, assumed to be Newtonian. However, less discrepancy is expected in large blood vessels.
\end{abstract}

\section{Introduction}

Le sang possède un quadruple rôle, (1) de transport d'espèces chimiques vers les organes cibles, (2) de régulation de la température, du $\mathrm{pH}$ et de la balance hydroélectrolytique, (3) de défense contre les corps étrangers et (4) de prévention des hémorragies par coagulation. La troisième fonction implique, en particulier, l'amarrage de lymphocytes sur la face mouillée de l'endothélium vasculaire puis la traversée de la paroi. Un phénomène similaire participe à la constitution des plaques athéromateuses. Le stade initial de l'athérogénèse est caractérisé par l'accumulation

(*) Auteur auquel doit être adressée la correspondance (e-mail : Marc.Thiriet@inria.fr)

$\left.{ }^{* *}\right)$ URA CNRS 343

(C) Les Éditions de Physique 1996 
de lipoprotéines dans la couche sous-endothéliale, lorsque le flux entrant n'est plus compensé par le flux sortant. Ces molécules sont ensuite capturées par les macrophages circulants, attirés dans la paroi, qui vont former des cellules spumeuses.

La relation entre l'athérogénèse et l'écoulement sanguin n'est plus à démontrer. Le transport de masse dans la lumière des vaisseaux physiologiques, le dépôt des particules convectées sur la paroi et leur transfert à travers la paroi, en raison de la complexité de chaque processus, ne peuvent être traités simultanément. En mécanique des fluides, l'investigation est focalisée sur les lieux de dépôts, à la lumière des valeurs que peut prendre localement la contrainte de cisaillement. Les dépôts de particules convectées sont, en effet, préférentiellement localisés dans les régions où le gradient de la vitesse à la paroi est faible, c'est-à-dire lorsque le temps de résidence à proximité de la paroi est plus long, comme, par exemple, à la frontière d'un décollement. Ainsi, le mécanisme dépendant du taux de cisaillement repose sur le transport de masse entre le sang et la paroi, contrairement à un processus induit par la contrainte de cisaillement, où l'action directe de forces sur la paroi est impliquée. Ce dernier mécanisme peut cependant affecter le dépôt de particules convectées.

Le sang peut être considéré comme une suspension concentrée d'hématies, de concentration ou hématocrite $\mathrm{Ht} \sim 45 \%$ chez l'homme adulte. La relation entre le taux de cisaillement $\dot{\gamma}$ et la viscosité relative $\mu$ (rapport entre la viscosité apparente de la suspension, i.e. la viscosité qu'aurait un fluide newtonien s'écoulant avec le même débit sous l'action d'un même gradient de pression, et la viscosité du solvant, libre de particules, dans les mêmes conditions physiques, qui est de 1,6 mPa s environ) d'une suspension d'hématies dans du plasma a été donnée par Chien (1970). Le comportement rhéofluidifiant du sang est observé pour des valeurs du taux de cisaillement $\sim 10^{-2} \leq \dot{\gamma} \leq \sim 10^{2} \mathrm{~s}^{-1}\left(\mu_{0} \leq \mu \leq \mu_{\infty}\right)$. En dehors de cet intervalle, le comportement du sang est supposé newtonien. Lorsqu'un échelon de cisaillement est appliqué à une suspension d'hématies, le passage d'un état stable à un autre s'accompagne d'une modification de structure de la suspension, qui dépend du temps : l'agrégation des hématies conduit aux fortes valeurs de la viscosité dans le domaine des faibles taux de cisaillement, la déformation et l'orientation des hématies expliquent les faibles valeurs de la viscosité aux plus forts taux de cisaillement [1].

L'hypothèse de fluide newtonien a été testée dans un modèle de tube rectiligne, de coude (angle de courbure de 90 degrés, rapport de courbure de 1/10), et d'embranchement plan symétrique (angle de bifurcation de 70 degrés, rapport des sections de 0,8 [2]). La méthode des éléments finis, caractérisée par la plus grande facilité à employer des conditions aux limites variées et la possibilité de travailler avec une géométrie complexe, est une méthode parfaitement adaptée à l'étude des écoulements physiologiques. L'objet de cette étude est de comparer les champs de vitesse d'un fluide dont le comportement rhéofluidifiant, similaire à celui du sang, est supposé être décrit par un modèle newtonien généralisé, et d'un fluide newtonien ayant la viscosité $\mu_{\infty}$, de l'ordre de 3 à $4 \mathrm{mPa}$ s, prise comme référence lorsque le sang est supposé newtonien. L'hématocrite est supposé constant. Des tests numériques ont été également effectués dans ces conduites avec la valeur $\mu_{0}$, dix fois plus grande que la valeur $\mu_{\infty}$.

\section{Méthode}

L'approximation numérique de la solution des équations de conservation s'appuie sur une formulation variationnelle de l'équation de Navier-Stokes:

$$
\left.\begin{array}{c}
\frac{\mathrm{d}}{\mathrm{d} t}(\mathbf{u}, \mathbf{v})+B(\mathbf{u}, \mathbf{v})+T(\mathbf{u} ; \mathbf{u}, \mathbf{v})+B^{\prime}(\mathbf{v}, p)=\langle l, \mathbf{v}\rangle, \\
\forall \mathbf{v} \in V \subset H^{1}(\Omega)^{3}
\end{array}\right\}
$$




$$
B^{\prime}(\mathrm{u}, q)=0, \forall q \in Q \subset L^{2}(\Omega)
$$

où $(\mathbf{u}, \mathbf{v})$ est le produit scalaire associé à l'espace $L^{2}(\Omega), B$ et $B^{\prime}$ des formes bilinéaires (pour la famille de conditions aux limites donnée par Conca et al. (1995), $B(\mathbf{u}, \mathbf{v})=\frac{1}{R e}[(\nabla \times \mathbf{u}, \nabla \times$ $\left.\mathbf{v})_{L^{2}(\Omega)}+(\nabla \cdot \mathbf{u}, \nabla \cdot \mathbf{v})_{L^{2}(\Omega)}\right]$, et $\left.B^{\prime}=-(\nabla \cdot \mathbf{v}, p)\right), T$ une forme trilinéaire $(T(\mathbf{u} ; \mathbf{u}, \mathbf{v})=$ $[(\mathbf{u} \cdot \nabla) \mathbf{u}] \cdot \mathbf{v}),<l, \mathbf{v}>$ le produit de dualité.

L'espace fonctionnel $V$ est défini de la manière suivante :

$$
V \quad\left\{\mathbf{v} \in H^{1}(\Omega)^{3},\left.\mathbf{v}\right|_{\Gamma_{1}}=0,\left.(\mathbf{v} \times \hat{\mathbf{n}})\right|_{\Gamma_{2}, \Gamma_{3}}=0, p\left(\Gamma_{2}\right)=\left.p\right|_{\Gamma_{2}}, p\left(\Gamma_{3}\right)=\left.p\right|_{\Gamma_{3}}\right\}
$$

$\left\{\Gamma_{2}\right\}_{1}^{3}$ étant une partition de la frontière $\Gamma$ du domaine de tluide $\Omega: \Gamma_{1}$ est la paroi sur laquelle est appliquée la classique condition d'adhérence, du type Dirichlet homogène, $\Gamma_{2}$ et $\Gamma_{3}$ sont les frontières d'entrée et de sortie de l'écoulement dans le domaine étudié.

La technique d'approximation retenue est une méthode d'éléments finis mixte $P_{1}$ bulle $/ P_{1}$ proposée par Arnold et al. (1984). Le domaine fluide est discrétisé par une famille de tétraèdres associés à une approximation $P_{1}$ pour la pression et $P_{1}$ bulle pour la vitesse. La vitesse est approchée par les éléments d'un espace constitué de fonctions continues dont la restriction à chaque simplexe s'écrit comme la somme d'une fonction affine du premier degré et d'un polynôme de degré 4 s'annulant sur les faces du tétraèdre (fonction bulle), exprimés à partir des coordonnées barycentriques $\left\{\lambda_{2}\right\}_{1}^{4}$.

$$
V_{\mathrm{h}}=\left\{v_{\mathrm{h}} \in H^{1}(\Omega), \forall K \in T_{\mathrm{h}},\left.v_{\mathrm{h}}\right|_{K} \in P(K)^{3}\right\},
$$

avec $V_{\mathrm{h}}$ : espace des fonctions $P^{1}$-bulle, et $P(K)=P^{1} \oplus S p\left(\prod \lambda_{2}\right)$. La pression est approchée par les éléments d'un deuxième espace de dimension finie composé de fonctions affines $P^{1}$ dans chaque élément fini.

$$
Q_{\mathrm{h}}=\left\{q_{\mathrm{h}} \in H^{1}(\Omega), \forall K \in T_{\mathrm{h}},\left.q_{\mathrm{h}}\right|_{K} \in P^{1}\right\},
$$

$Q_{\mathrm{h}}$ : espace des fonctions continues $P^{1}$.

Le système matriciel linéaire à deux inconnues $\mathbf{u}_{\mathrm{h}}$ et $p_{\mathrm{h}}$ issu des équations discrétisées par un schéma d'éléments finis mixtes est résolu à l'aide de l'algorithme d'Uzawa, couplé à une méthode de gradient conjugué préconditionné. Le système d'équations algébriques est initialisé en résolvant le problème de Stokes, avec les mêmes conditions aux limites. Le terme convectif non-linéaire de l'équation de Navier-Stokes est traité par la méthode des caractéristiques [5].

2.1. GÉomÉTRIE DES MODÈLES. - Le coude et l'embranchement sont considérés comme des modèles élémentaires d'artères. Trois domaines ont été employés, un tube droit, pour tester le code de calcul, un coude, et une bifurcation. Les caractéristiques du coude sont les suivantes : un angle de courbure de 90 degrés, un rapport de courbure (entre le rayon du tube et le rayon de courbure de l'axe du coude $R / R_{\mathrm{c}}$ égal à $1 / 10$. Il est précédé d'un tube rectiligne, de longueur $L=7 R$ et suivi d'un conduit droit $(L=16 R)$. Le domaine est discrétisé en 48000 tétraèdres (9000 nœuds).

La géométrie de l'embranchement est, en règle générale, assez complexe. La ramification du vaisseau géniteur s'accompagne d'une modification progressive de l'aire de la section droite, définissant une région intermédiaire appelée zone de transition. Le calcul de la surface de contour de la zone de transition est effectué à l'aide de fonctions spline cubiques, grâce à l'algorithme de Catmull [2]. Les caractéristiques géométriques de la bifurcation plane symétrique sont présentées dans le tableau I. 
Tableau I. - Caractéristiques géométriques de la bifurcation symétrique. Le domarne est discrétisé en 36400 tétraèdres (7300 nœuds).

[Geometry of the symmetrical planar bifurcation. The fluid domain is discretized into 36400 tetrahedra (7300 nodes).]

\begin{tabular}{|l|l|c|}
\hline Tube principal & longueur & $1 d_{\mathrm{p}}$ \\
\hline \multirow{3}{*}{ Zone de transition } & longueur & $1 d_{\mathrm{p}}$ \\
& rayon de courbure & $0,7 d_{\mathrm{p}}$ \\
& angle de bifurcation & $70^{\circ}$ \\
\hline Branche de soustie & diamètre & $d_{\mathrm{b}}=0,625 d_{\mathrm{p}}$ \\
& longueur & $10 d_{\mathrm{b}}$ \\
\hline
\end{tabular}

2.2. Modèle RHÉologique. - En présence d'un fluide complexe, tel que le sang, le problème consiste à trouver l'expression appropriée du tenseur des extra-contraintes $\underline{\underline{\sigma}}$, second terme du tenseur des contraintes de Cauchy. Dans le cas d'un fluide supposé obéir au modèle newtonien généralisé, cette expression est donnée par $\underline{\underline{\sigma}}=2 \mu(\dot{\gamma}, T) \underline{d}(\underline{u})$, où $\underline{u}$ est la vitesse, $T$ la température, $\underline{\underline{d}}=(1 / 2)\left(\underline{\underline{\nabla u}}+\underline{\underline{\nabla u}}{ }^{T}\right)$ le tenseur taux de déformation, $\dot{\gamma}=\imath_{2}(\underline{\underline{d}})$ le taux de cisaillement $\left(\imath_{2}\right.$. second invariant $)$.

Le modèle de Carreau est, a priori, bien adapté pour décrire le comportement du sang, puisqu'il produit une valeur de viscosité de faible taux de cisaillement $\mu_{0}$, une valeur de viscosité aux forts taux de cisaillement $\mu_{\infty}$ et une loi de puissance aux taux de cisaillement intermédiaires :

$$
\frac{\mu(\dot{\gamma})-\mu_{\infty}}{\mu_{o}-\mu_{\infty}}=\frac{1}{\left(1+(\lambda \dot{\gamma})^{2}\right)^{p}}
$$

$\lambda$ représente le taux de cisaillement à mi-pente et $p$ la pente du régime rhéofluidifiant. Les valeurs retenues pour les diverses variables de la loi de Carreau sont les suivantes:

$$
\mu_{0} \sim 40 \mathrm{mPa} \mathrm{s}^{-1}, \quad \mu_{\infty} \sim 4 \mathrm{mPa} \mathrm{s}^{-1} \lambda \sim 0,25 \mathrm{~s}, \quad \mathrm{p} \sim 1 / 3 .
$$

La valeur de $p$ sélectionnée est inférieure au seuil imposé par la théorie développée par Baranger et al. (1990). En outre, dans un écoulement laminaire stationnaire établi dans un tube rectiligne, le produit de la viscosité par le taux de cisaillement est proportionnel à la distance radiale, le coefficient de proportionnalité étant la moitié du gradient de pression axial. Pour obtenir le taux de cisaillement, il faut inverser la relation ; le terme $\mu(\dot{\gamma}(r)) \dot{\gamma}(r)$ doit varier de façon monotone en fonction de $\dot{\gamma}$, ce qui a été vérifié pour $p<1 / 2$. La valeur de l'exposant $p=1 / 3$ évite les problèmes de divergence et de bifurcation de la méthode numérique.

La méthode de résolution numérique de l'équation de Navier-Stokes pour un fluide au comportement newtonien généralisé est basée sur le modèle de Smagorinsky (1963), qui consiste à ajouter un terme d'échange turbulent :

$$
\frac{\partial \mathbf{u}}{\partial t}+(\mathbf{u} \cdot \nabla) \mathbf{u}-\nabla \cdot \underline{\underline{\tau}}(\mathbf{u}, p)=-\nabla \cdot \underline{\underline{R}}, \operatorname{sur} \Omega \times[0, T]
$$

avec $\underline{\underline{R}} \sim-0,01 h^{2}\|\underline{\underline{d}}(\mathbf{u})\| \underline{\underline{d}}(\mathbf{u})$ et $\underline{\underline{\tau}}=-p \underline{\underline{I}}+2 \mu(\mathbf{u}) \underline{\underline{d}}(\mathbf{u})$. Dans le cas d'un fluide nonnewtonien, le coefficient effectif d'échañge est la somme du terme habituel de viscosité sanguine 
$\mu_{\infty}$ et d'un terme dépendant du taux de cisaillement en chaque point du domaine, donné par la loi de Carreau.

$$
\underline{\underline{R}}=\frac{\mu_{0}-\mu_{\infty}}{\left(1+\lambda^{2}\|\underline{\underline{d}}(\mathbf{u})\|^{2}\right)^{p}} \underline{\underline{d}}(\mathbf{u}),
$$

\section{Résultats}

3.1. ÉCOULEMENT laminaIRe STATIONNAIRE DANS UN TUBE RECTILIGNE. - La viscosité du fluide de comportement rhéofluidifiant est toujours plus grande que celle du sang supposé newtonien, de viscosité $\mu_{\infty}$. Le fluide s'écoule donc plus difficilement : le gradient de pression longitudinal nécessaire au déplacement du fluide de viscosité $\mu(\dot{\gamma})$ est a priori plus grand que le gradient de pression longitudinal d'un écoulement de fluide newtonien. Ce fait est confirmé par le calcul ; le gradient de pression dans le fluide de viscosité $\mu(\dot{\gamma})$ est environ huit fois plus élevé que le gradient de pression dans le fluide de viscosité $\mu_{\infty}$.

Il est bien connu que les effets non-newtoniens se traduisent par un aplatissement du profil de la vitesse dans la zone centrale de la lumière du tube, où le taux de cisaillement est faible voire nul. La zone à faible taux de cisaillement est, en effet, plus large pour le fluide de viscosité $\mu(\dot{\gamma})$ : la région où le cisaillement est plus petit que $1,85 \mathrm{~s}^{-1}$ est de $0,05 R$ plus large, pour un nombre de Reynolds de 250 , valeur considérée de manière à obtenir un écoulement établi en sortie du tube. Par contre, le taux de cisaillement à proximité de la paroi $(0,94 \leq r / R \leq 1)$ atteint des valeurs plus élevées de $15 \%$.

Les champs de pression et de vitesse dans la zone d'entrée du tube droit ressemblent davantage à ceux calculés pour un fluide newtonien de viscosité $\mu_{0}$. Dans l'hypothèse du modèle newtonien généralisé, et avec les valeurs prises, le sang peut être considéré comme un fluide newtonien de viscosité apparente environ huit fois plus grande que la valeur $\mu_{\infty}$ de référence.

3.2. ÉCOULEMENT DANS UN COUDE. - Le fluide est soumis à une force d'inertie centrifuge supplémentaire, qui agit perpendiculairement à la direction locale de l'axe du tube. La répartition asymétrique par rapport à l'axe du tube de la quantité de mouvement impose une nouvelle distribution de la pression transversalement. Le mouvement des particules de fluide est caractérisé par une trajectoire hélicoïdale. À tout instant et en tout lieu du domaine fluide, le vecteur vitesse est décomposé en une composante parallèle à la direction locale de l'axe du tube et une composante perpendiculaire, dans le plan de la section droite. Cette dernière composante constitue le vecteur vitesse de l'écoulement secondaire.

Le champ de vitesse de l'écoulement de fluide de viscosité $\mu(\dot{\gamma})$ et le champ de vitesse du tluide de viscasité $\mu_{\infty}$ sont très différents, pour un rapport entre les forces d'inertie convective et les forces visqueuses donné, quelque soit la valeur du nombre de Reynolds, ou du nombre de Dean, la géométrie étant fixée. Le pic de vitesse axiale est situé à une distance beaucoup plus grande de la paroi externe (Fig. 1). Les effets inertiels sont d'autant moins impliqués que le fluide est plus visqueux. L'écoulement secondaire du fluide non-newtonien est moins intense que l'écoulement secondaire du fluide de viscosité $\mu_{\infty}$. De plus, le champ de vitesse du fluide non-newtonien tend plus rapidement vers un profil établi. Il est plus proche du champ de vitesse du fluide newtonien de viscosité $\mu_{0}$ (Tab. III). Cependant, le champ de vitesse principal du fluide de viscosité $\mu_{0}$ est davantage centré sur l'axe du coude. Ainsi, la viscosité apparente du sang est plus grande que la valeur $\mu_{\infty}$ de référence.

La différence de pression axiale atteint des valeurs environ trois fois plus élevées que celles du gradient de pression du fluide de viscosité $\mu_{\infty}$. Le gradient de pression transversal est par contre plus petit, l'effet de la courbure pour un nombre de Reynolds donné étant plus faible. 


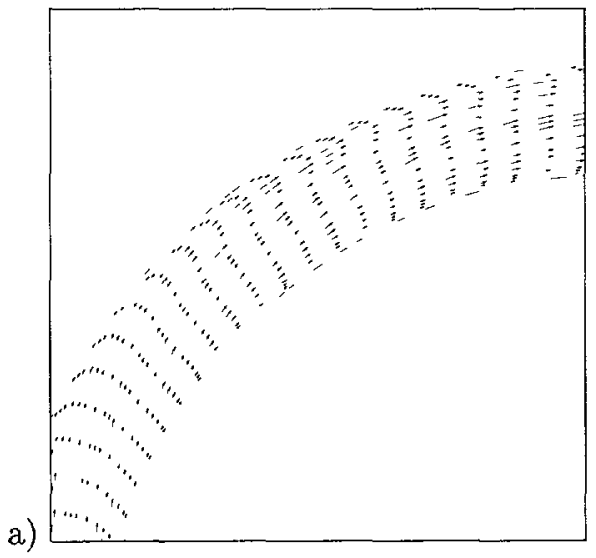

b)
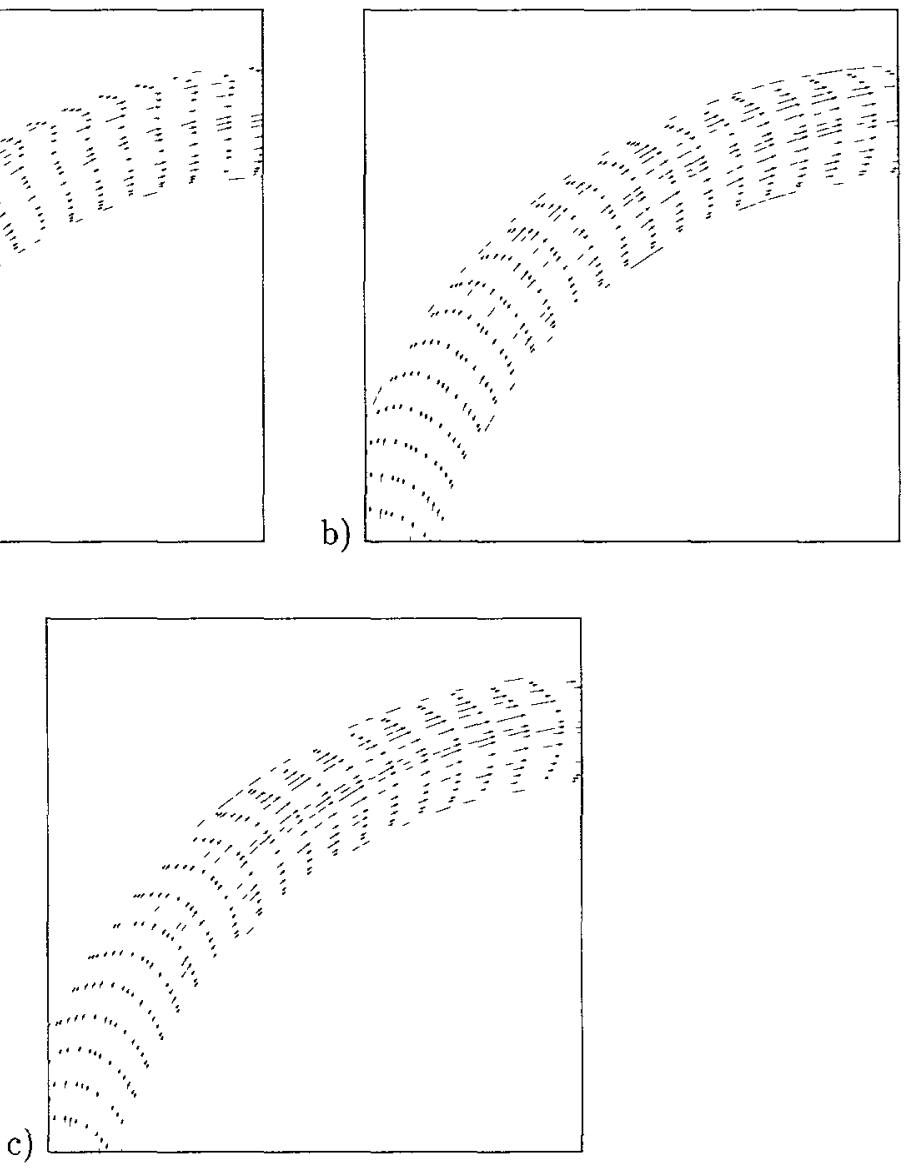

Fig. 1. - Champ de vitesse dans le plan de courbure d'un coude (angle de 90 degrés, rapport de courbure de $1 / 10$ ) pour un nombre de Reynolds Re de 500 (nombre de Dean de 160) et trois types de fluide, dont deux newtoniens, a) et b), avec des valeurs de viscosité qui correspondent aux extrema de la. viscosité du fluide non-newtonien c), $\mu_{\infty}$ (palier aux forts taux de cisaillement $\dot{\gamma}>10^{2} \mathrm{~s}^{-1}$ de la loi de comportement rhéologique du sang) et $\mu_{0}$ (valeur aux très faibles taux de cisaillement $\dot{\gamma}<10^{-2} \mathrm{~s}^{-1}$ ), dix fois plus élevée. Le profil de la composante axiale de la vitesse d'un fluide de viscosité $\mu_{\infty}$ est caractérisé par un fort gradient de vitesse près de la paroi externe du coude et, outre la région du maximum de vitesse, par une plage de taux de cisaillement très faibles dans le cœur de l'écoulement, davantage située dans la partie interne (bande d'épaisseur $0,2 R$ située de part et d'autre du plan de courbure). L'écoulement de fluide de viscosité $\mu_{0}$ ou ayant un comportement rhéofluidifiant, est moins perturbé par la singularité.

[Velocity profiles in the curvature plane of a curved pipe (curvature angle of 90 degrees, curvature ratio of 1/10), computed for three types of fluid (Reynolds number of 500, Dean number of 160). a), b) Newtonian fluids with viscosity $\mu_{\infty}$ and $\mu_{0}$ respectively. c) generalized Newtonian fluid of shearthinning type with viscosity $\mu(\dot{\gamma})$ varying between two extrema $\mu_{\infty}$ (reference value) and $\mu_{0}$, according to the Carreau law. The flow field of the Newtonian fluid of reference (viscosity $\mu_{\infty}$ ) is characterized by a high shear rate near the outer bend and a core region, slightly skewed toward the inner edge, with zero shear rate. The flow of both the Newtonian fluid (viscosity $\mu_{0}$ ) and shear-thinning fluid is much less disturbed than the reference fluid flow by the duct curvature; the inertia forces are overwhelmed by the viscous forces.] 
Tableau II. - Valeurs maximales du taux de cisaillement dans le coude quand le nombre de Reynolds est égal à 500 ou 1000 (nombre de Dean de 160 et 320). Le taux de cisaillement croît des sections proximales aux sections distales. Le maximum est situé près de la paroi externe, quelque soit la viscosité du fluide. Le taux de cisaillement du fluide de viscosité $\mu(\dot{\gamma})$ est plus faible que le taux de cisaillement du fluide newtonien de viscosité $\mu_{\infty}$, qui définit l'état de référence, sauf sur la paroi interne; les valeurs sont semblables ou un peu plus fortes que celles du fluide newtonien calculées avec la viscosité $\mu_{0}$.

[Maximal shear-rate values in the bend for flow Reynolds numbers Re of 500 and 1000 (Dean number of 160 and 320). The shear rate increases from the bend entry to its distal segment. Maxima are located on the outer edge for the three types of fluid. The shear rate in the nonNewtonian fluid $\mu(\dot{\gamma})$ is lower than the shear rate in the Newtonian fluid with viscosity $\mu_{\infty}$ (reference value), except near the inner bend; it is similar or slightly higher than the shear rate in the newtonian fluid with viscosity $\mu_{0}$.]

\begin{tabular}{|l|l||c|}
\hline $\begin{array}{l}\text { Nombre de } \\
\text { Reynolds }\end{array}$ & Viscosité & $\begin{array}{c}\dot{\gamma}_{\max } \\
\left(\mathrm{s}^{-1}\right)\end{array}$ \\
\hline \multirow{3}{*}{500} & $\mu_{\infty}$ & 63 \\
& $\mu(\dot{\gamma})$ & 35 \\
& $\mu_{0}$ & 25 \\
\hline \multirow{3}{*}{1000} & $\mu_{\infty}$ & 145 \\
& $\mu(\dot{\gamma})$ & 69 \\
& $\mu_{0}$ & 64,5 \\
\hline
\end{tabular}

Le gradient de pression longitudinal dans le fluide de viscosité $\mu_{0}$ est plus important que le gradient du fluide de viscosité $\mu(\dot{\gamma})$.

Dans le coude, le taux de cisaillement $\dot{\gamma}$ dans le fluide de viscosité $\mu_{\infty}$ diminue très vite sur la paroi interne pour devenir à peu près constant sur tout le coude. Par contre, le cisaillement sur la paroi externe croît jusque dans le tronçon distal du coude. Le taux de cisaillement est beaucoup plus faible lorsque la viscosité augmente, sauf sur la paroi interne, puisque la distribution de la vitesse tend à devenir uniforme. Le maximum du taux de cisaillement augmente en fonction du nombre de Reynolds, mais moins fortement lorsque le fluide est non-newtonien (Tab. II).

En résumé, le comportement du fluide de viscosité $\mu(\dot{\gamma})$ en écoulement stationnaire laminaire dans un coude est voisin, mais non semblable de celui du fluide newtonien dont la viscosité, est égale à la valeur de la viscosité aux faibles taux de cisaillement $\left(\mu_{0}\right)$. Cependant, aucune valeur caractéristique du taux de cisaillement dans l'écoulement rhéofluidifiant ne peut être déduite à partir d'entités caractéristiques de l'écoulement de fluide newtonien.

3.3. ÉCOULEMENT DANS UN EMBRANCHEMENT PLAN SYMÉTRIQUE. - La plupart des caractéristiques de l'écoulement dans la branche, en dehors d'un court tronçon d'entrée, résulte de l'effet de courbure du conduit. La division du tube principal n'influe cependant pas seulement sur l'écoulement par la courbure de la paroi. Elle met en place les conditions nécessaires à l'apparition, dès que les forces d'inertie sont suffisamment élevées, du décollement observé à l'entrée des tubes latéraux. Les différences notables par rapport au coude étudié sont : (1) l'existence d'un point d'impact, zone de forte pression (1'apex), (2) l'angle de courbure de la paroi externe de l'embranchement de 55 degrés, (3) les aires de section droite du tronc $A_{\mathrm{t}}$ et de la branche 
Tableau III. - Distance en norme $L^{2}(\Omega)$ entre les champs de vitesse du fluide newtonien, de viscosité $\mu_{\infty}$ ou $\mu_{0}$ et du fluide de viscosité $\mu(\dot{\gamma})$, pour des nombres de Reynolds 500 et 1000. La valeur du champ de vitesse du fluide non-newtonien est beaucoup plus proche de celle du champ de vitesse du fiuide newtonien en écoulement avec un nombre de Reynolds 10 fois plus faible, particulièrement lorsque le nombre de Reynolds est élevé. La norme indiquée est une norme relative. rapportée à la distance entre les valeurs des champs de la vitesse pour les viscosités $\mu(\dot{\gamma})$ et $\mu_{\infty}$ et pour un nombre de Reynolds de 500 .

[Distance in $L^{2}(\Omega)$ norm between the velocity fields of a Newtonian fluid (viscosity $\mu_{\infty}$ or $\mu_{0}$, ten times higher than $\mu_{\infty}$ ) and a generalized Newtonian fluid in a symmetrical bifurcation for flow Reynolds numbers Re of 500 and 1000 (Dean number of 160 and 320). The difference in velocity fields is much less between the non-Newtonian fluid and the Newtonian fluid of viscosity $\mu_{0}$ than between the non-Newtonian fluid and the Newtonian fluid of viscosity $\mu_{\infty}$, which defines, for $\mathrm{Re}=500$, the reference.]

\begin{tabular}{|cc|c|c|}
\hline & & $\mu_{\infty}$ & $\mu_{0}$ \\
\hline$\mu(\dot{\gamma})$, & $\operatorname{Re}=500$ & 1,0 & 0,18 \\
$\mu(\dot{\gamma})$, & $\operatorname{Re}=1000$ & 3,86 & 0,35 \\
\hline
\end{tabular}

Tableau IV. - Distance en norme $L^{2}(\Omega)$ entre les champs de la vitesse d'un fluide newtonien, de viscosité $\mu_{\infty}$ ou $\mu_{0}$ et d'un fluide de viscosité $\mu(\dot{\gamma})$ (comportement rhéofluidifiant), en écoulement dans une bifurcation symétrique plane avec des nombres de Reynolds 500 et 1000.

[Distance in $L^{2}(\Omega)$ norm between the velocity fields of a Newtonian fluid (viscosity $\mu_{\infty}$ or $\mu_{0}$ ) and a generalized newtonian fluid of shear-thinning type in a symmetrical bifurcation for flow Reynolds number of 500 and 1000 (Dean number of 160 and 320).]

\begin{tabular}{|cc|c|c|}
\hline & & $\mu_{\infty}$ & $\mu_{0}$ \\
\hline$\mu(\dot{\gamma})$, & $\operatorname{Re}=500$ & 1,0 & 0,27 \\
$\mu(\dot{\gamma})$, & $\operatorname{Re}=1000$ & 5,0 & 1,6 \\
\hline
\end{tabular}

$A_{\mathrm{b}}$ différentes (rapport $A_{\mathrm{b}} / A_{\mathrm{t}}$ égal à 0,4 ), et (4) la présence d'un convergent-divergent (zone de transition).

Dans la bifurcation, aucun décollement n'est visible pour un nombre de Reynolds $\operatorname{Re}<2000$. les forces visqueuses étant trop grandes, alors que pour $\mathrm{Re} \geq 800$, les zones de recirculation sont observées dans la région d'entrée des branches lorsque le fluide est newtonien (viscosité $\mu_{\infty}$ ). En aval, l'écoulement est établi plus rapidement. Le champ de vitesse du fluide de viscosité $\mu(\dot{\gamma})$ est, comme dans les deux autres domaines, plus proche du champ de vitesse calculé avec la viscosité $\mu_{0}$ (Tab. IV). Le gradient de pression longitudinal est plus élevé dans le cas nonnewtonien, mais le gradient transversal reste plus faible. Le taux de cisaillement dans le fluide non-newtonien est beaucoup plus faible que le taux de cisaillement dans le fluide newtonien (viscosité $\mu_{\infty}$ ) sur la paroi interne et plus élevé sur la paroi externe des branches (Fig. 2). 


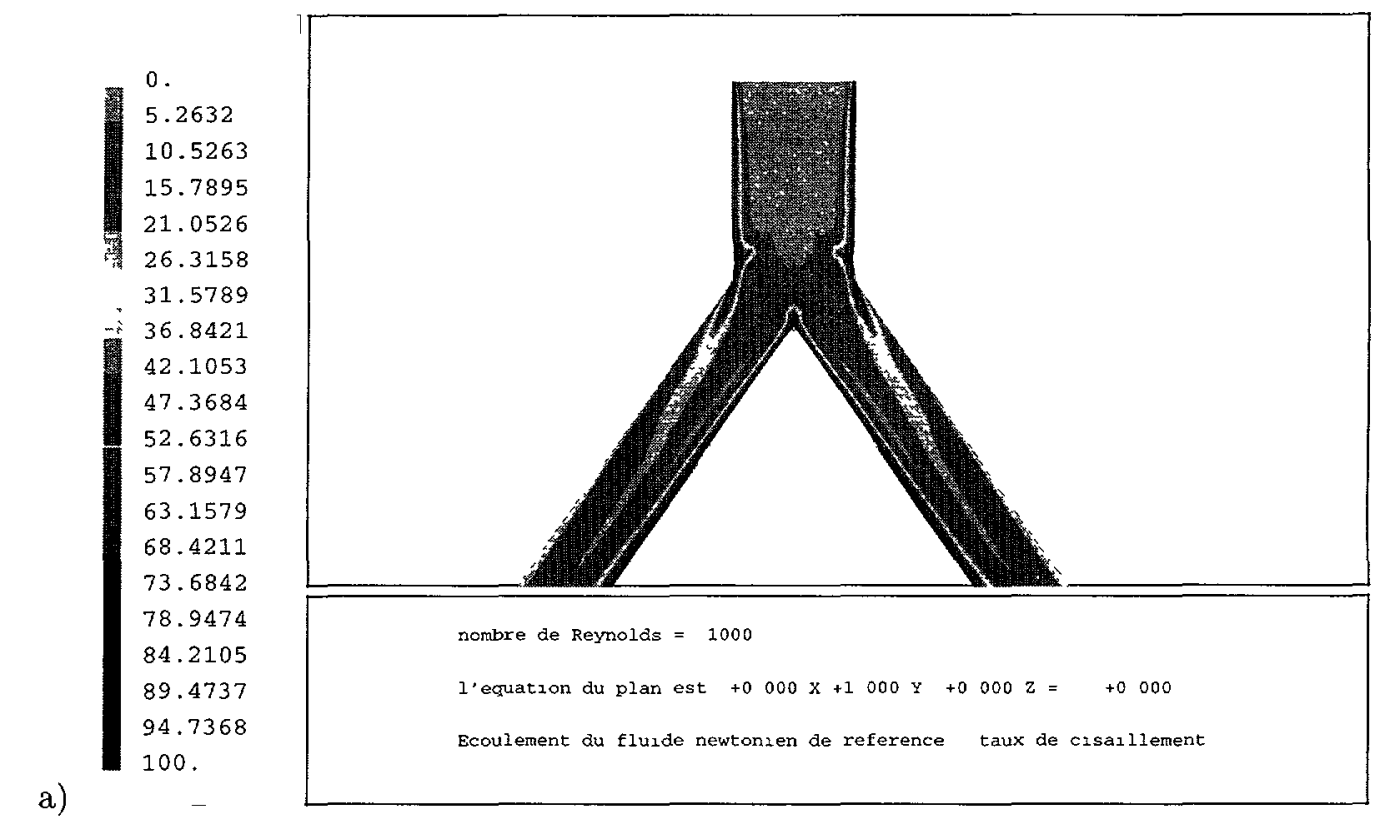

a)

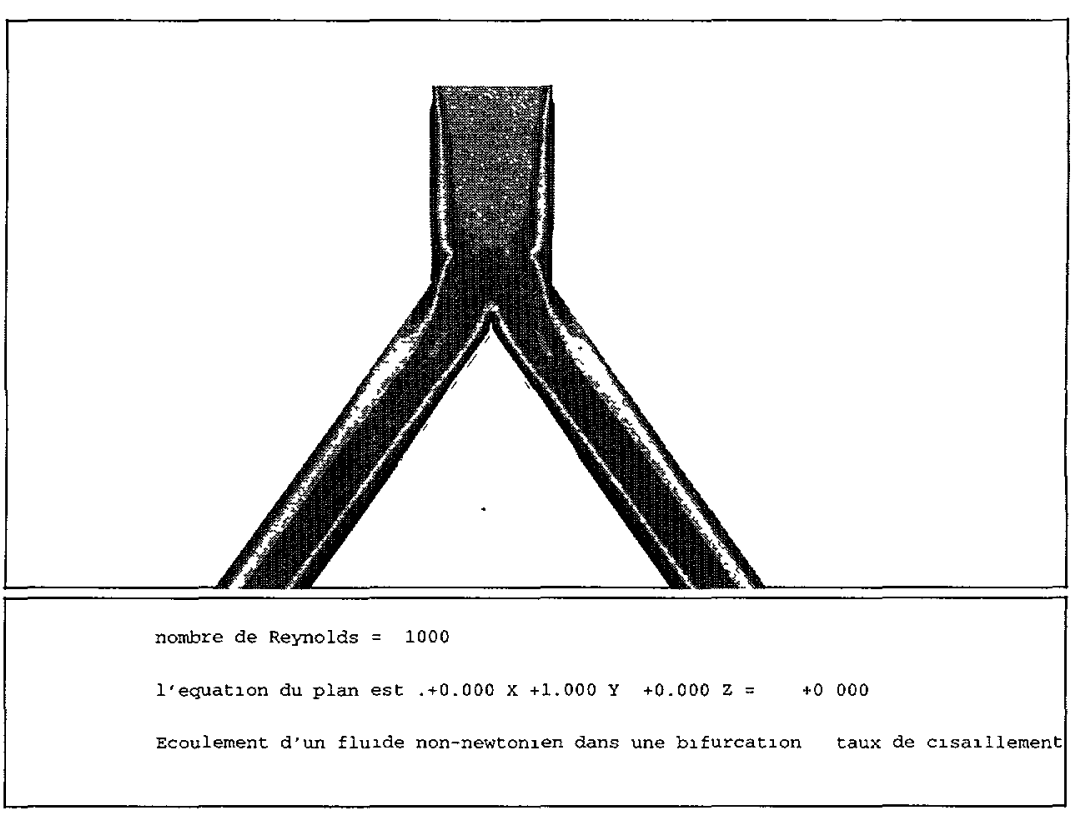

Fig. 2. - Taux de cisaillement dans la bifurcation pour un nombre de Reynolds de 1000 (nombre de Dean de 320). a) Fluide newtonien de viscosité $\mu_{\infty}$; b) comportement rhéofluidifiant. Une zone de recirculation apparaît le long de la paroi externe de la région d'entrée de la branche. Lorsque le comportement du fluide obéit à la loi de Carreau, le décollement n'est plus visible. Le taux de cisaillement dans le fluide rhéofluidifiant est beaucoup plus élevé que le taux de cisaillement du fluide newtonien sur la paroi externe (paroi la plus proche du centre de courbure).

[Shear rate in a planar symmetrical bifurcation (Reynolds number of 1000, Dean number of 320). a) Newtonian fluid (vistosity $\mu_{\infty}$ ). b) shear-thinning fluid. Flow separation appears in the Newtonian fluid flow in the entrance segment of the branch. When the constitutive law of the fluid is described by the Carreau model, the fluid runs alongside the outer bifurcation wall, along which the shear rate is higher than in the reference Newtonian fluid case.] 
Tableau V. - Les trois classes de cellules sanguines. Les globules rouges ont un rôle prédominant sur le comportement rhéologique du sang.

[The three kinds of blood cells. The red blood cells play a major role on the blood rheological behaviour.]

\begin{tabular}{|c|c|c|c|c|c|}
\hline $\begin{array}{c}\text { cellules } \\
\text { sanguines }\end{array}$ & $\begin{array}{c}\text { quantité } \\
\left(\mathrm{mm}^{-3}\right)\end{array}$ & $\begin{array}{c}\text { proportion } \\
\text { relative }\end{array}$ & taille & $\begin{array}{c}\text { volume } \\
\text { cellulaire } \\
\left(\mu \mathrm{m}^{3}\right)\end{array}$ & $\begin{array}{c}\text { pourcentage } \\
\text { du volume } \\
\text { cellulaire } \\
\text { total }\end{array}$ \\
\hline hématies & $5 \times 10^{6}$ & 1 & $\sim 8$ & $\sim 90$ & $97 \%$ \\
\hline leucocytes & $5 \times 10^{3}$ & $10^{-3}$ & $\sim 8-20$ & $\sim 1400$ & $2 \%$ \\
\hline thrombocytes & $3 \times 10^{5}$ & $6 \times 10^{-2}$ & $\sim 3$ & $\sim 10$ & $1 \%$ \\
\hline
\end{tabular}

\section{Discussion}

4.1. GÉOMÉTRIE DES MODÈLES. - L'extrême variabilité de géométrie du vaisseau chez un sujet donné, en fonction de l'environnement biochimique, et entre les individus, rend illusoire tout modèle représentant plus ou moins parfaitement l'élément du réseau de conduites sélectionné, qui ne peut correspondre qu'à une configuration temporaire chez un sujet sélectionné. Face à la diversité des dimensions possibles, le choix de la configuration des modèles se porte vers une géométrie simple mais représentative; elle prend en compte la forme et les dimensions les plus fréquemment observées. Ainsi, la stratégie des travaux biomécaniques est basée sur le principe du modèle. Il s'agit, non pas de décrire quantitativement les champs des grandeurs dynamiques dans une configuration figée du milieu vivant, mais de fournir des informations qualitatives permettant de comprendre le comportement du système circulatoire. Le réseau artériel est caractérisé par la présence de nombreux coudes et d'embranchements. Le coude peut être considéré comme l'unité de base. Tout embranchement peut, en première approximation, être assimilé à une juxtaposition de deux tubes courbes, avec une condition de glissement sur la partie commune de la frontière. L'écoulement dans la branche est en effet similaire au courant de fluide dans un coude. Cependant, l'écoulement dans la bifurcation possède des caractéristiques propres : écoulement secondaire particulier (de type puits-sources) dans la zone de transition [2], décollement dans la région d'entrée des branches. Coudes et embranchements sont, par conséquent, des modèles complémentaires ; ils ont servi de domaines tests.

4.2. HYPothÈSES. - Le sang est une suspension concentrée de cellules, de macromolécules biologiques et d'électrolytes. Huit à douze heures après un repas, le sang est composé d'environ $80 \mathrm{~g}$ d'eau libre et de $20 \mathrm{~g}$ de matériaux solides pour $100 \mathrm{ml}$ de sang. Il existe trois classes de cellules sanguines ; la plus importante est celle des globules rouges, dont la concentration habituelle par unité de volume ou hématocrite $\mathrm{Ht}$ est d'environ $45 \%$ chez l'homme adulte. Le sang est donc considéré comme une suspension concentrée d'hématies. En effet, le nombre d'hématies par unité de volume excède par un facteur $10^{3}$ environ le nombre de leucocytes, ce qui permet de négliger le rôle des globules blancs sur le comportement rhéologique du sang. Quant aux plaquettes, elles sont dix fois moins nombreuses et de taille plus petite (cf. Tab. V).

Sur une échelle de longueur grande devant la taille du globule rouge, le sang peut être considéré comme un milieu continu. Les équations de conservation considérées dans ce travail sont 
donc appropriées. Le fluide devient inhomogène lorsque les propriétés physiques de la suspension sont discontinues à l'échelle habituelle du milieu continu. Certes, la répartition des cellules en suspension cisaillée n'est pas uniforme dans la section droite du vaisseau. Néanmoins, le rapport entre le diamètre de la particule sphérique équivalente et celui du vaisseau étant très faible $\left(\mathcal{O}\left(10^{-5}\right)\right)$, la suspension concentrée, qui s'écoule à grande vitesse, est supposée homogène. En outre, l'existence d'une stratification due à la présence d'une couche de "lubrification", complètement dépourvue de cellules, est peu probable ; l'ordre de grandeur du rapport entre l'épaisseur de la couche, à proximité de la paroi, caractérisée par une déplétion de cellules et le rayon hydraulique du vaisseau est de $10^{-4}$. L'hypothèse de fluide homogène peut être retenue. Par contre, le caractère non-newtonien du sang devrait a priori être pris en compte dans les gros vaisseaux. En effet, le comportement viscoélastique du sang ne peut être négligé lorsque le taux de cisaillement est faible, comme dans certaines régions des coudes et des embranchements.

4.3. Modèle RhÉologique. - Lorsque la relation entre le taux de cisaillement et la contrainte de cisaillement du fluide est linéaire, une seule variable physique suffit à la décrire; la viscosité dynamique du fluide newtonien est constante dans des conditions ambiantes données. Plusieurs variables physiques doivent être prises en compte quand le comportement n'est plus linéaire, y compris lorsque les propriéteś physico-chimiques du milieu sont fixées, la viscosité apparente $\mu_{\text {app }}$ du sang dépendant de la microstructure de la suspension et donc des divers agents susceptibles de la modifier. Cette structure est affectée, en particulier, par l'hématocrite $\mathrm{Ht}$ et les contraintes cinétiques. La viscosité du sang dépend enfin de l'histoire des déformations, mais ce facteur est généralement négligé. Une revue des modèles mathématiques de la relation taux de cisaillement en fonction de la contrainte de cisaillement, avec comme paramètres l'hématocrite, parfois la viscosité dynamique du plasma sanguin et trois ou quatre variables d'ajustement aux mesures, est fournie par Easthorpe et Brooks (1980). Aucune de ces lois n'est satisfaisante. Des modèles rhéologiques classiques ont été adaptés au comportement du sang. Le modèle de Casson [8] $\tau^{1 / 2}=\tau_{Y}^{1 / 2}+\mu_{\mathrm{C}} \dot{\gamma}^{1 / 2}$ fut le plus employé comme loi de comportement rhéologique du sang, avec comme paramètres caractéristiques la viscosité dite de Casson $\mu_{\mathrm{C}}=\left[\mu_{\mathrm{pl}} /(1-\mathrm{Ht})^{\mathrm{k}-1}\right]^{1 / 2}(k$ est une constante déterminée à partir des données expérimentales [9]) et le seuil de contrainte apparent $\tau_{\mathrm{Y}} \sim 10 \mathrm{~m} \mathrm{~Pa}$ [10]). L'inconvénient de cette loi réside dans l'existence de la contrainte seuil ; en outre, même si ce modèle est meilleure que la loi de puissance, il ne reflète pas le comportement viscoélastique du sang. Le modèle phénoménologique de Cross (1965), pour une suspension concentrée $(\phi<0,6)$, peut servir à décrire le comportement du sang : $\mu(\dot{\gamma})=\mu_{\infty}+\frac{\left(\mu_{o}-\mu_{\infty}\right)}{1+(\lambda \dot{\gamma})^{p}}$, où $\lambda$ représente l'inverse du taux de cisaillement à mi-pente et $p$ la pente de la relation entre les deux paliers. Dans le cas du sang, $p \sim 2$. Le modèle de Cross n'est qu'une forme particulière du modèle de Carreau.

La relation entre le taux de cisaillement et la viscosité calculée par la loi de Carreau a été comparée à des courbes expérimentales obtenues à partir du sang prélevé sur des hommes sains non-fumeurs (Fig. 3).

Les mesures sont effectuées le plus souvent à l'état stable, dans des conditions standards (température, $\mathrm{pH}$, concentration en fibrinogène, hématocrite, .. ). Les valeurs crêtes sont parfois retenues. En général, les données sont obtenues à l'aide de viscosimétres à cylindres coaxiaux. Les courbes $\dot{\gamma}-\mu$ varient d'un individu à l'autre. La variabilité intra-sujet est également très importante, en raison des propriétés physico-chimiques du sang qui changent d'une période à l'autre de la journée. Les relations donnant la contrainte de cisaillement d'une part et la viscosité d'autre part, pour des données d'entrée et une géométrie du domaine fluide fixées, sont connues lorsque le fluide est newtonien. Les valeurs des taux de cisaillement apparents mesurés dépendant de l'appareillage et du modèle expérimental qui lui est associé (hypothèses, 


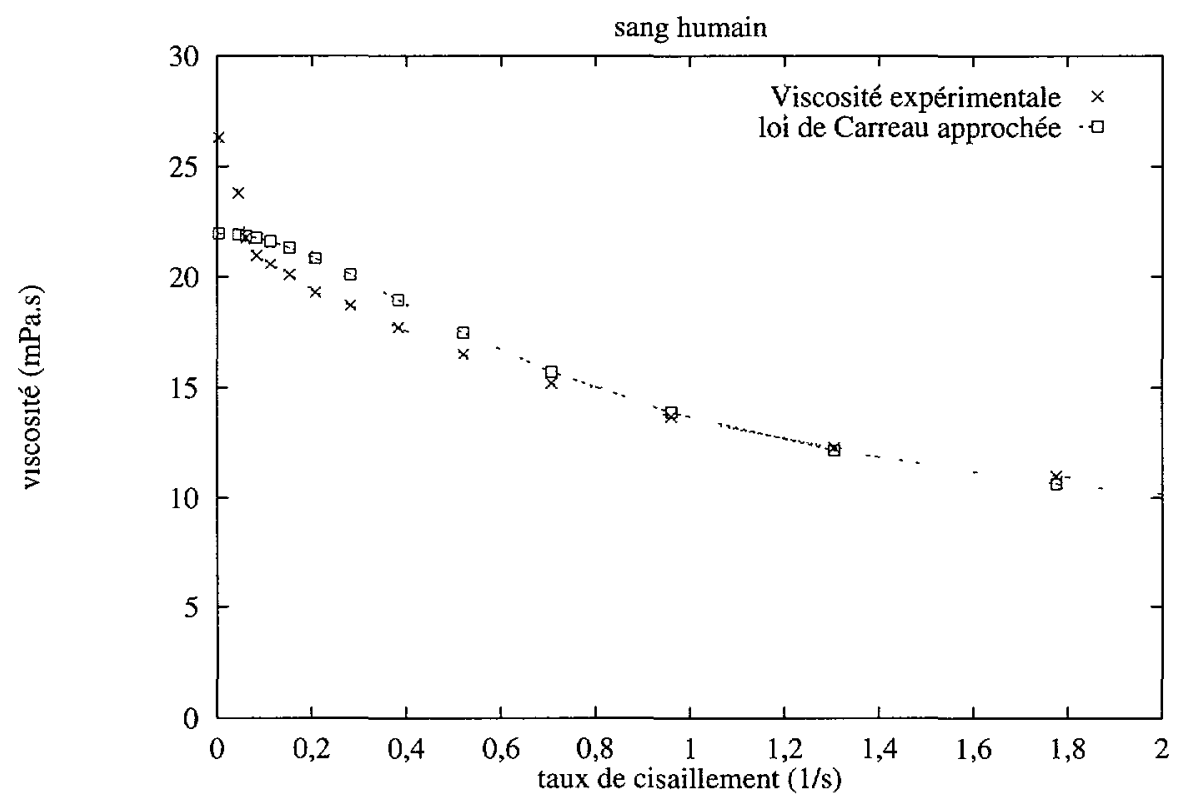

\begin{tabular}{|c|c||c|c|}
\hline $\begin{array}{c}\text { Cisaillement } \\
\left(\mathrm{s}^{-1}\right)\end{array}$ & $\begin{array}{c}\text { Viscosité } \\
\mathrm{mPa} \mathrm{s}\end{array}$ & $\begin{array}{c}\text { Cisaillement } \\
\left(\mathrm{s}^{-1}\right)\end{array}$ & $\begin{array}{c}\text { Viscosité } \\
(\mathrm{mPa} \mathrm{s})\end{array}$ \\
\hline 0,0328 & 26,3 & 3,28 & 8,8 \\
0,0446 & 23,8 & 4,46 & 8 \\
0,0606 & 21,8 & 6,06 & 7,2 \\
0,0825 & 21,0 & 8,25 & 6,6 \\
0,112 & 20,6 & 11,2 & 6,1 \\
0,152 & 20,1 & 15,2 & 5,6 \\
0,207 & 19,3 & 20,7 & 5,3 \\
0,281 & 18,7 & 28,1 & 5 \\
0,382 & 17,7 & 38,2 & 4,7 \\
0,519 & 16,5 & 51,9 & 4,5 \\
0,707 & 15,2 & 70,7 & 4,4 \\
0,960 & 13,7 & 96,0 & 4 \\
1,30 & 12,3 & 131 & 3,9 \\
1,77 & 11 & 177,5 & 3,7 \\
2,41 & 9,9 & 241 & 3,6 \\
\hline
\end{tabular}

Fig. 3. - Relation $\mu-\dot{\gamma}$ expérimentale et loi de Carreau ajustée. La Loi de Carreau approche très bien la relation expérimentale pour les cisaillements élevés. Par contre, l'adaptation aux valeurs expérimentales, peu fiables, aux faibles taux de cisaillement, n'est pas bonne. Les valeurs des paramètres de la loi de Carreau sont : $\mu_{0}=22 \mathrm{mPa} \mathrm{s}, \mu_{\infty}=3,6 \mathrm{mPa} \mathrm{s}, \lambda=2,1 \mathrm{~s}, p=0,35$. Les valeurs de la relation entre la viscosité du sang d'un individu mâle sain de 56 ans non fumeur et le taux de cisaillement sont données dans le tableau ci-dessus.

[Experimental shear rate versus viscosity relationship and fitted Carreau law. The experimental data couples, from a non-smoking healthy male subject, 56 years of age, are given in the above table. A good agreement is obtained for shear-rate values in medium and high ranges. However, there is a discrepancy between the two sets of values at low shear rate, the experimental ones being much less reliable. The parameters of the Carreau model are the following: $\mu_{0}=22 \mathrm{mPas}, \mu_{\infty}=3,6 \mathrm{mPas}, \lambda=2,1 \mathrm{~s}, p=0,35$.] 
fluide étalon, .. ) un certain degré d'imprécision sur la détermination des valeurs des grandeurs cinématiques en résulte lorsque le comportement n'est plus newtonien. Les erreurs inhérentes à toute expérimentation, l'imprécision sur les taux de glissement apparents, et, enfin, l'extrême variabilité intra- et inter-sujet des données biologiques collectées, limitent la portée de la relation rhéologique quantitative. En conséquence, les valeurs des paramètres de la relation entre la viscosité et le taux de cisaillement sont celles habituellement admises, la qualité de la relation taux de cisaillement en fonction de la viscosité étant jugé adéquate. La démarche suivie ne cherche pas à refléter un aspect limité de la réalité, mais à analyser, à l'aide d'un modèle, un mécanisme pour comprendre, d'ailleurs non immuablement, la réalité. Les conclusions dépendent des données d'entrée et des hypothèses faites.

4.4. IMPLiCATIONS ET LIMITES DES RÉSultats. - Dans la littérature, la valeur $\mu_{\infty}\left(\mu(\dot{\gamma})>10^{2} \mathrm{~s}^{-1}\right)$ est prise pour la viscosité du sang, lorsqu'elle est supposée indépendante du taux de cisaillement. Cependant, nos résultats montrent que la viscosité newtonienne apparente du sang est toujours entre $\mu_{\infty}$ et $\mu_{0}\left(\mu(\dot{\gamma})<10^{-2} \mathrm{~s}^{-1}\right.$ ), dix fois plus grande que $\mu_{\infty}$. L'écoulement de fluide de viscosité élevée dans les gros troncs artériels semble moins sensible aux singularités du réseau de conduites lorsque le fluide a un comportement non-newtonien, de même que pour un fluide newtonien fortement visqueux. Dans les coudes, les taux de cisaillement à la paroi sont plus forts pour un fluide rhéofluidifiant sur la courbure interne et légérement plus faibles sur la courbure externe; ainsi, les particules se déposent plus difficilement sur la paroi interne. Dans la bifurcation, les taux de cisaillement sont également beaucoup plus fort sur la paroi externe des branches ; la disparition du décollement est un autre élément défavorable au dépôt de particules. Le comportement rhéologique du sang ne favoriserait donc pas l'apparition de plaques d'athérome ou de thrombose.

Le modèle rhéologique adopté possède un certain nombre de limitations. Le fluide a un comportement newtonien généralisé ; le rôle des déformations subies antérieurement par le fluide sur son comportement est ignoré. Le modèle ne reflète donc qu'imparfaitement le comportement rhéologique du sang. En outre, les valeurs des paramètres de la loi de comportement reposent sur des données expérimentales. Rappelons que la relation entre la viscosité et le taux de cisaillement, observée expérimentalement est expliquée par la déformabilité des globules rouges aux forts taux de cisaillement et par l'agrégation des hématies aux faibles taux de cisaillement [1]. L'hématie est convectée dans les artères sans subir de grandes déformations, contrairement au transport dans les capillaires. De plus, à la sortie du cœur, la présence d'agrégats d'hématies est peu probable. Le transit rapide des hématies dans les artères de gros calibre ne favorise pas l'agrégation, dont la constante de temps n'est pas négligeable. Puisque les deux phénomènes, déformation et agrégation, sont responsables du comportement rhéofluidifiant observé dans des conditions de mesure précises et sans lien avec la circulation artérielle, la viscosité de la suspension concentrée sanguine oscille-t-elle toujours entre deux valeurs différentes d'un facteur dix ? Les mesures ont été effectuées dans des conditions stationnaires à l'aide d'un viscosimètre à cylindres coaxiaux, dont l'entrefer était de $0,96 \mathrm{~mm}$. De telles conditions s'apparentent davantage à celles rencontrées dans la microcirculation. Or, la nature de l'écoulement sanguin y est très différente de celle observées dans les vaisseaux de gros calibre. Enfin, l'équipe de Chien (1970) a montré qu'une suspension de globules rouges transformés $(\mathrm{Ht}=45 \%)$, qui ne peuvent ni se déformer ni s'agréger, se comporte comme un fluide newtonien. Il est néanmoins vraisemblable que le comportement rhéologique du sang s'écarte de celui d'un fluide newtonien. Les effets sont cependant moins marqués que les résultats obtenus, avec des extrema de la viscosité très différents. 


\section{Conclusion}

La valeur $\mu_{\infty} \sim 4 \mathrm{~s}^{-1}$ est prise comme viscosité de référence lorsque le sang est supposé newtonien. Cependant, nos résultats montrent que l'écoulement tridimensionnel d'un fluide au comportement rhéofluidifiant possède des caractéristiques beaucoup plus proches de celles de l'écoulement d'un fluide newtonien de viscosité $\mu_{0}$, correspondant à la valeur de la viscosité du sang aux faibles taux de cisaillement, que de celles de l'écoulement du sang supposé newtonien, de viscosité $\mu_{\infty}$.

La viscosité apparente du sang prend une valeur dans l'intervalle $\left[\mu_{\infty}, \mu_{0}\right]$. Le principe de la méthode expérimentale, qui fournit la valeur de $\mu_{0}$, est basé sur un cisaillement du sang dans des conditions éloignées de celles de la circulation dans les grandes artères. Nos résultats doivent donc être pondérés par le fait que la valeur $\mu_{0}$ puisse être moins forte que la valeur considérée dans ce travail. L'écart de comportement avec le fluide newtonien de viscosité $\mu_{\infty}$ serait ainsi moins important.

\section{Remerciements}

Ce travail a bénéficié de l'aide de l'INSERM (contrat N 910914). Nous remercions J.C. Lelièvre et les rapporteurs pour leurs commentaires.

\section{Bibliographie}

[1] Chien S., Shear dependence of effective cell volume as a determinant of blood viscosity, Science 168 (1970) 977-978.

[2] Thiriet M., Pares C., Saltel E. et Hecht F., Numerical simulation of steady flow in a model of the aortic bifurcation, ASME J. Biomech. Eng. 114 (1992) 40-49.

[3] Conca C., Pares C., Pironneau O. et Thiriet M., A computational model of Navier-Stokes equations with imposed pressure and velocity fluxes, Int. J. Numer. Methods in Fluids 20 (1995) 267-287.

[4] Arnold D., Brezzi F. et Fortin M., A stable finite element for the Stokes equations, Calcolo 21 (1984) 337-344.

[5] Pironneau $\mathrm{O}$., On the transport-diffusion algorithm and its applications to the NavierStokes equations, Numer. Math. 38 (1982) 309-322.

[6] Smagorinski J.F., General circulation model of the atmosphere, Mon. Weath. Rev. 91 (1963) 99-164.

[7] Easthope P.L. et Brooks D.E., A comparison of rheological constitutive functions for whole human blood, Biorheol. 17 (1980) 235-247.

[8] Casson N., "Rheology of disperse systems", Mill D.C., Ed. (Pergamon Press, 1959) pp. 84-102.

[9] Perktold K., Resch M. et Peter R.O., Pulsatile non-Newtonian flow characteristics in a three-dimensional human carotid bifurcation model, ASME J. Biomech. Eng. 113 (1991) 464-475.

[10] Charm S.E. et Kurland G.S., Viscometry of human blood for shear rates of 0-100000 1/s, Nature 206 (1965) 617-618.

[11] Baranger J. et Najib K., Analyse numérique des écoulements quasi-newtoniens dont la Viscosité obéit à la loi puissance ou la loi de Carreau, Num. Math. 58 (1990) 34-49. 